\title{
Analytical Method for Computation of Phase-Detector Characteristic
}

\author{
G. A. Leonov, N. V. Kuznetsov, M. V. Yuldashev, and R. V. Yuldashev
}

\begin{abstract}
Discovery of undesirable hidden oscillations, which cannot be found by simulation, in models of phase-locked loop (PLL) showed the importance of development and application of analytical methods for the analysis of such models. Approaches to a rigorous nonlinear analysis of analog PLL with multiplier phase detector (classical PLL) and linear filter are discussed. An effective analytical method for computation of multiplier/mixer phase-detector characteristics is proposed. For various waveforms of high-frequency signals, new classes of phase-detector characteristics are obtained, and dynamical model of PLL is constructed.
\end{abstract}

Index Terms-Analog integrated circuits, nonlinear analysis, phase-detector characteristic, phase-locked loop (PLL).

\section{INTRODUCTION}

D ISCOVERY OF undesirable hidden oscillations [1], which cannot be found by simulation, in phase-locked loop (PLL) models [2] showed the importance of development and application of analytical methods for the analysis of such models. To carry out the nonlinear analysis of PLL, it is necessary to consider PLL models in signal and phase-frequency spaces [3]-[6]. For constructing an adequate nonlinear mathematical model of PLL in phase-frequency space, it is necessary to find the characteristic of phase detector (PD) (PD is a nonlinear element used in PLL to match tunable signals). The PD inputs are high-frequency signals of reference and tunable oscillators, and the output contains a low-frequency error correction signal, corresponding to a phase difference of input signals. For the suppression of high-frequency component at PD output (if such component exists), a low-pass filter is applied. The characteristic of PD is a function defining a dependence of signal at the output of PD (in the phase-frequency space) on the phase difference of signals at the input of PD. PD characteristic depends on the realization of PD and waveforms of input signals.

The characteristics of classical PD-multiplier for typical sinusoidal signal waveforms are well known to engineers [3], [7]-[10].

Furthermore, following [11], on the examples of PD in the form of multiplier, the general principles of computing the PD characteristics for various types of signals, based on a rigorous

Manuscript received April 4, 2012; revised June 9, 2012; accepted August 4, 2012. Date of publication September 14, 2012; date of current version October 12, 2012. This work was supported in part by the Academy of Finland, by the Ministry of Education and Science, by Saint Petersburg State University, and by Russian Foundation of Basic Research (Russia). This brief was recommended by Associate Editor S. Levantino.

G. A. Leonov is with the Saint Petersburg State University, Russia.

N. V. Kuznetsov, M. V. Yuldashev, and R. V. Yuldashev are with the Saint Petersburg State University, Russia, and also with the University of Jyväskylä, Finland (e-mail: nkuznetsov239@gmail.com).

Digital Object Identifier 10.1109/TCSII.2012.2213362

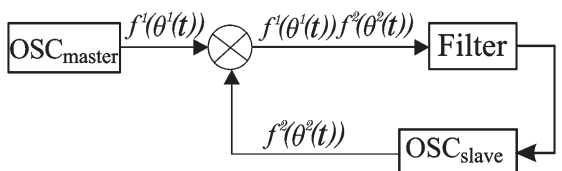

Fig. 1. Block diagram of PLL on the level of electronic realization.

mathematical analysis of high-frequency oscillations [12], [13], will be considered.

\section{Description of Classical PLL in Signal Space}

Consider classical PLL on the level of electronic realization (Fig. 1).

Here, $\mathrm{OSC}_{\text {master }}$ is a master oscillator, and $\mathrm{OSC}_{\text {slave }}$ is a slave oscillator [tunable voltage-control oscillator (VCO)], which generate oscillations $f^{p}(t)=f^{p}\left(\theta^{p}(t)\right), p=1,2$ with $\theta^{p}(t)$ as phases, correspondingly.

The block $\otimes$ is a multiplier (used as PD) of oscillations $f^{1}(t)$ and $f^{2}(t)$, and the signal $f^{1}(t) f^{2}(t)$ is its output. The relation between the input $\xi(t)$ and the output $\sigma(t)$ of linear filter is as follows:

$$
\sigma(t)=\alpha_{0}(t)+\int_{0}^{t} \gamma(t-\tau) \xi(\tau) d \tau
$$

where $\gamma(t)$ is an impulse response function of filter and $\alpha_{0}(t)$ is an exponentially damped function depending on the initial data of filter at moment $t=0$. By assumption, $\gamma(t)$ is a differentiable function with bounded derivative (this is true for the most considered filters [9]).

\section{A. High-Frequency Property of Signals}

Suppose that the waveforms $f^{1,2}(\theta)$ are bounded $2 \pi$-periodic piecewise differentiable functions ${ }^{1}$ (this is true for the most considered waveforms). Consider Fourier series representation of such functions

$$
\begin{aligned}
f^{p}(\theta) & =\sum_{i=1}^{\infty}\left(a_{i}^{p} \sin (i \theta)+b_{i}^{p} \cos (i \theta)\right), \quad p=1,2 \\
a_{i}^{p} & =\frac{1}{\pi} \int_{-\pi}^{\pi} f^{p}(\theta) \sin (i \theta) d \theta \quad b_{i}^{p}=\frac{1}{\pi} \int_{-\pi}^{\pi} f^{p}(\theta) \cos (i \theta) d \theta .
\end{aligned}
$$

A high-frequency property of signals can be reformulated in the following way. By assumption, the phases $\theta^{p}(t)$ are smooth functions (this means that frequencies are changing continuously, which is corresponding to classical PLL analysis

\footnotetext{
${ }^{1}$ The functions with a finite number of jump discontinuity points differentiable on their continuity intervals.
} 


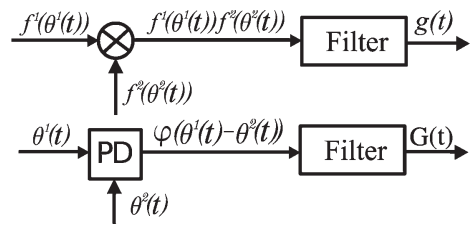

Fig. 2. Multiplier and filter; PD and filter.

[9], [10]). Suppose also that there exists a sufficiently large number $\omega_{\min }$ such that the following conditions are satisfied on the fixed time interval $[0, T]$ :

$$
\dot{\theta}^{p}(\tau) \geq \omega_{\min }>0, \quad p=1,2
$$

where $T$ is independent of $\omega_{\text {min }}$ and $\dot{\theta}^{p}(t)$ denotes frequencies of signals. The frequencies difference is assumed to be uniformly bounded

$$
\left|\dot{\theta}^{1}(\tau)-\dot{\theta}^{2}(\tau)\right| \leq \Delta \omega, \quad \forall \tau \in[0, T]
$$

Requirements (2) and (3) are obviously satisfied for the tuning of two high-frequency oscillators with close frequencies [9], [10]. Let us introduce $\delta=\omega_{\min }^{-(1 / 2)}$. Consider the relations

$$
\begin{aligned}
\left|\dot{\theta}^{p}(\tau)-\dot{\theta}^{p}(t)\right| & \leq \Delta \Omega, \quad p=1,2 \\
|t-\tau| & \leq \delta, \quad \forall \tau, t \in[0, T]
\end{aligned}
$$

where $\Delta \Omega$ is independent of $\delta$ and $t$. Conditions (2)-(4) mean that the functions $\dot{\theta}^{p}(\tau)$ are almost constant and the functions $f^{p}\left(\theta^{p}(\tau)\right)$ are rapidly oscillating on small intervals $[t, t+\delta]$.

The boundedness of derivative of $\gamma(t)$ implies

$$
|\gamma(\tau)-\gamma(t)|=O(\delta) \quad|t-\tau| \leq \delta, \quad \forall \tau, t \in[0, T] .
$$

\section{Phase-Detector Characteristic Computation}

Consider the two block diagrams shown in Fig. 2. Here, PD is a nonlinear block with characteristic $\varphi(\theta)$. The phases $\theta^{p}(t)$ are PD block inputs, and the output is a function $\varphi\left(\theta^{1}(t)-\theta^{2}(t)\right)$. The PD characteristic $\varphi(\theta)$ depends on the waveforms of input signals $f^{p}(\theta)$.

The signal $f^{1}\left(\theta^{1}(t)\right) f^{2}\left(\theta^{2}(t)\right)$ and the function $\varphi\left(\theta^{1}(t)-\right.$ $\left.\theta^{2}(t)\right)$ are the inputs of the same filters with the same impulse response function $\gamma(t)$ and with the same initial state. The outputs of filters are functions $g(t)$ and $G(t)$, respectively. By (1), one can obtain $g(t)$ and $G(t)$

$$
\begin{aligned}
g(t) & =\alpha_{0}(t)+\int_{0}^{t} \gamma(t-\tau) f^{1}\left(\theta^{1}(\tau)\right) f^{2}\left(\theta^{2}(\tau)\right) d \tau \\
G(t) & =\alpha_{0}(t)+\int_{0}^{t} \gamma(t-\tau) \varphi\left(\theta^{1}(\tau)-\theta^{2}(\tau)\right) d \tau .
\end{aligned}
$$

Then, using the approaches outlined in [11] and [14]-[16], the following result can be proved.

Theorem 1: Let conditions (2)-(5) be satisfied and

$\varphi(\theta)=\frac{1}{2} \sum_{l=1}^{\infty}\left(\left(a_{l}^{1} a_{l}^{2}+b_{l}^{1} b_{l}^{2}\right) \cos (l \theta)+\left(a_{l}^{1} b_{l}^{2}-b_{l}^{1} a_{l}^{2}\right) \sin (l \theta)\right)$.
Then, the following relation:

$$
|g(t)-G(t)|=O(\delta), \quad \forall t \in[0, T]
$$

is valid.

Proof: Suppose that $t \in[0, T]$. Consider the difference

$$
\begin{array}{r}
g(t)-G(t)=\int_{0}^{t} \gamma(t-s)\left[f^{1}\left(\theta^{1}(s)\right) f^{2}\left(\theta^{2}(s)\right)\right. \\
\left.-\varphi\left(\theta^{1}(s)-\theta^{2}(s)\right)\right] d s .
\end{array}
$$

Suppose that there exists $m \in \mathbb{N} \cup\{0\}$ such that $t \in[m \delta,(m+$ 1) $\delta$ ]. By definition of $\delta$, we have $m<(T / \delta)+1$. The continuity condition implies that $\gamma(t)$ is bounded on $[0, T]$ and $f^{1}(\theta)$ and $f^{2}(\theta)$ are bounded on $\mathbb{R}$. Since $f^{1,2}(\theta)$ are piecewisedifferentiable, one can obtain

$$
a_{i}^{1,2}=O\left(\frac{1}{i}\right) \quad b_{i}^{1,2}=O\left(\frac{1}{i}\right) .
$$

Hence, $\varphi(\theta)$ converges uniformly, and $\varphi(\theta)$ is continuous, piecewise differentiable, and bounded. Then, the following estimates:

$$
\begin{gathered}
\int_{t}^{(m+1) \delta} \gamma(t-s) f^{1}\left(\theta^{1}(s)\right) f^{2}\left(\theta^{2}(s)\right) d s=O(\delta) \\
\int_{t}^{(m+1) \delta} \gamma(t-s) \varphi\left(\theta^{1}(s)-\theta^{2}(s)\right) d s=O(\delta) .
\end{gathered}
$$

are satisfied. It follows that (8) can be represented as

$$
\begin{aligned}
& g(t)-G(t)=\sum_{k=0}^{m} \int_{[k \delta,(k+1) \delta]} \gamma(t-s) \\
& \quad \times\left[f^{1}\left(\theta^{1}(s)\right) f^{2}\left(\theta^{2}(s)\right)-\varphi\left(\theta^{1}(s)-\theta^{2}(s)\right)\right] d s+O(\delta) .
\end{aligned}
$$

Prove now that, on each interval $[k \delta,(k+1) \delta]$, the corresponding integrals are equal to $O\left(\delta^{2}\right)$.

Condition (5) implies that, on each interval $[k \delta,(k+1) \delta]$, the following relation:

$\gamma(t-s)=\gamma(t-k \delta)+O(\delta), \quad t>s ; \quad s, t \in[k \delta,(k+1) \delta]$.

is valid. Here, $O(\delta)$ is independent of $k$, and the relation is satisfied uniformly with respect to $t$. By (10), (11), and the boundedness of $f^{1}(\theta), f^{2}(\theta)$, and $\varphi(\theta)$, it can be obtained that

$$
\begin{aligned}
& g(t)-G(t)=\sum_{k=0}^{m} \gamma(t-k \delta) \int_{[k \delta,(k+1) \delta]} \\
& \times\left[f^{1}\left(\theta^{1}(s)\right) f^{2}\left(\theta^{2}(s)\right)-\varphi\left(\theta^{1}(s)-\theta^{2}(s)\right)\right] d s+O(\delta) .
\end{aligned}
$$

Denote

$$
\theta_{k}^{p}(s)=\theta^{p}(k \delta)+\dot{\theta}^{p}(k \delta)(s-k \delta), \quad p=1,2 .
$$

Then, for $s \in[k \delta,(k+1) \delta]$, condition (4) yields

$$
\theta^{p}(s)=\theta_{k}^{p}(s)+O(\delta) .
$$


From (3) and the boundedness of derivative $\varphi(\theta)$ on $\mathbb{R}$, it follows that

$$
\int_{[k \delta,(k+1) \delta]}\left|\varphi\left(\theta^{1}(s)-\theta^{2}(s)\right)-\varphi\left(\theta_{k}^{1}(s)-\theta_{k}^{2}(s)\right)\right| d s=O\left(\delta^{2}\right) .
$$

If $f^{1}(\theta)$ and $f^{2}(\theta)$ are continuous on $\mathbb{R}$, then, for $f^{1}\left(\theta^{1}(s)\right) f^{2}\left(\theta^{2}(s)\right)$, one obtains the following relation:

$$
\begin{aligned}
& \int_{[k \delta,(k+1) \delta]} f^{1}\left(\theta^{1}(s)\right) f^{2}\left(\theta^{2}(s)\right) d s \\
& \quad=\int_{[k \delta,(k+1) \delta]} f^{1}\left(\theta_{k}^{1}(s)\right) f^{2}\left(\theta_{k}^{2}(s)\right) d s+O\left(\frac{1}{\delta^{2}}\right) .
\end{aligned}
$$

Let us consider why this estimate is valid for the considered class of piecewise differentiable waveforms. Since the conditions (2) and (4) are satisfied and the functions $\theta^{1,2}(s)$ are differentiable and satisfy (3), for all $k=0, \ldots, m$, there exist sets $E_{k}$ [the union of sufficiently small neighborhoods of discontinuity points of $\left.f^{1,2}(t)\right]$ such that the following relation takes place: $\int_{E_{b}} d s=O\left(\delta^{2}\right)$, in which case the relation is satisfied uniformly with respect to $k$. Then, from the piecewise differentiability and the boundedness of $f^{1,2}(\theta)$, it can be proved (14).

By (14) and (13), relation (12) can be rewritten as

$$
\begin{aligned}
g(t)-G(t)= & \sum_{k=0}^{m} \gamma(t-k \delta) \int_{[k \delta,(k+1) \delta]} \\
& {\left[f^{1}\left(\theta_{k}^{1}(s)\right) f^{2}\left(\theta_{k}^{2}(s)\right)-\varphi\left(\theta_{k}^{1}(s)-\theta_{k}^{2}(s)\right)\right] d s+O(\delta) } \\
= & \sum_{k=0}^{m} \gamma(t-k \delta) \int_{[k \delta,(k+1) \delta]} \\
& {\left[\left(\sum_{i=1}^{\infty} a_{i}^{1} \cos \left(i \theta_{k}^{1}(s)\right)+b_{i}^{1} \sin \left(i \theta_{k}^{1}(s)\right)\right)\right.} \\
& \times\left(\sum_{j=1}^{\infty} a_{j}^{2} \cos \left(j \theta_{k}^{2}(s)\right)+b_{j}^{2} \sin \left(j \theta_{k}^{2}(s)\right)\right) \\
& \left.-\varphi\left(\theta_{k}^{1}(s)-\theta_{k}^{2}(s)\right)\right] d s+O(\delta) .
\end{aligned}
$$

Since the conditions (2)-(4) are satisfied, it is possible to choose $O(1 / \delta)$ of sufficiently small time intervals of length $O\left(\delta^{3}\right)$, outside of which the functions $f^{p}\left(\theta^{p}(t)\right)$ and $f^{p}\left(\theta_{k}^{p}(t)\right)$ are continuous. It is known that, on each interval, which has no discontinuity points, Fourier series of the functions $f^{1}(\theta)$ and $f^{2}(\theta)$ converge uniformly. Then, there exists a number $M=M(\delta)>$ 0 such that, outside sufficiently small neighborhoods of discontinuity points of $f^{p}\left(\theta^{p}(t)\right)$ and $f^{p}\left(\theta_{k}^{p}(t)\right)$, the sum of the first $M$ series terms approximates the original function with accuracy to $O(\delta)$. In this case, by relation (15) and the boundedness of $f^{1}(\theta)$ and $f^{2}(\theta)$ on $\mathbb{R}$, it can be obtained

$$
\begin{aligned}
g(t)-G(t) & =\sum_{k=0}^{m} \gamma(t-k \delta) \int_{[k \delta,(k+1) \delta]} \sum_{i=1}^{M} \sum_{j=1}^{M} \\
& \times\left[\mu_{i, j}(s)-\varphi\left(\theta_{k}^{1}(s)-\theta_{k}^{2}(s)\right)\right] d s+O(\delta)
\end{aligned}
$$

where

$$
\begin{aligned}
\mu_{i, j}(s)=\frac{1}{2}( & \left(a_{i}^{1} a_{j}^{2}+b_{i}^{1} b_{j}^{2}\right) \cos \left(i \theta^{1}-j \theta^{2}\right) \\
& +\left(-a_{i}^{1} b_{j}^{2}+b_{i}^{1} a_{j}^{2}\right) \sin \left(i \theta^{1}-j \theta^{2}\right) \\
& +\left(-b_{i}^{1} b_{j}^{2}+a_{i}^{1} a_{j}^{2}\right) \cos \left(i \theta^{1}+j \theta^{2}\right) \\
& \left.+\left(a_{i}^{1} b_{j}^{2}+b_{i}^{1} a_{j}^{2}\right) \sin \left(i \theta^{1}+j \theta^{2}\right)\right) .
\end{aligned}
$$

From definition of $\delta$ and (9), it follows that, $\forall i \in \mathbb{N}, j \in \mathbb{N}$

$$
\int_{[k \delta,(k+1) \delta]} \frac{1}{i} \cos \left(j\left(\omega_{\min } s+c_{0}\right)\right) d s=\frac{O\left(\delta^{2}\right)}{i j}
$$

is valid. Taking into account (17) and (2), one obtains the estimate

$$
\int_{[k \delta,(k+1) \delta]} b_{j}^{p} \cos \left(j \theta_{k}^{p}(s)\right) d s=\frac{O\left(\delta^{2}\right)}{j^{2}} .
$$

Similar estimate is also valid for the addends with sin.

Consider the addend involving $\cos \left(i \theta_{k}^{1}(s)+j \theta_{k}^{2}(s)\right)$ in $\mu_{i, j}(s)$. By (2), it can be obtained $i \dot{\theta}^{1}(k \delta)+j \dot{\theta}^{2}(k \delta) \geq(i+$ j) $\omega_{\min }$. Then, (17) yields the following relation:

$$
\begin{aligned}
& \int_{[k \delta,(k+1) \delta]} \cos \left(i\left(\theta^{1}(k \delta)+\dot{\theta}^{1}(k \delta)(s-k \delta)\right)\right. \\
& \left.+j\left(\theta^{2}(k \delta)+\dot{\theta}^{2}(k \delta)(s-k \delta)\right)\right) d s \\
& =\int_{[k \delta,(k+1) \delta]} \cos \left(\left(i \dot{\theta}^{1}(k \delta)+j \dot{\theta}^{2}(k \delta)\right) s+i \theta^{1}(k \delta)+j \theta^{2}(k \delta)\right. \\
& =O\left(\frac{\delta^{2}}{i+j}\right) .
\end{aligned}
$$

Then

$$
\begin{array}{r}
\sum_{i=1}^{M} \sum_{j=1}^{M} \int_{[k \delta,(k+1) \delta]} \frac{-b_{i}^{1} b_{j}^{2}+a_{i}^{1} a_{j}^{2}}{2} \cos \left(i\left(\theta_{k}^{1}(s)\right)+j\left(\theta_{k}^{2}(s)\right)\right) d s \\
=\sum_{i=1}^{M} \sum_{j=1}^{M} \frac{O\left(\delta^{2}\right)}{i j(I+j)} .
\end{array}
$$

The convergence of series $\sum_{i=1}^{\infty} \sum_{j=1}^{\infty}(1 / i j(i+j))$ implies that the above expression is $O\left(\delta^{2}\right)$. Obviously, a similar relation occurs for the addend $\sin \left(i \theta_{k}^{1}(s)+j \theta_{k}^{2}(s)\right)$.

Thus, by (16)

$$
\begin{gathered}
g(t)-G(t) \\
=\sum_{k=0}^{m} \gamma(t-k \delta) \int_{[k \delta,(k+1) \delta]} \\
{\left[\sum _ { i = 1 } ^ { M } \sum _ { j = 1 } ^ { M } \left(\frac{a_{i}^{1} a_{j}^{2}+b_{i}^{1} b_{j}^{2}}{2} \cos \left(i \theta_{k}^{1}(s)-j \theta_{k}^{2}(s)\right)\right.\right.} \\
\left.+\frac{a_{i}^{1} b_{j}^{2}-b_{i}^{1} a_{j}^{2}}{2} \sin \left(i \theta_{k}^{1}(s)-j \theta_{k}^{2}(s)\right)\right) \\
\left.-\varphi\left(\theta_{k}^{1}(s)-\theta_{k}^{2}(s)\right)\right] d s+O(\delta) .
\end{gathered}
$$


Note that, here, the addends with indices $i=j$ give, in sum, $\varphi\left(\theta_{k}^{1}(s)-\theta_{k}^{2}(s)\right)$ with accuracy to $O(\delta)$. Consider the addends with indices $i<j$, involving cos (for the addends with indices $i>j$, involving sin, similar relations are satisfied). By (3), similar to (18), the following relation:

$$
\begin{aligned}
& \sum_{i=2}^{M} \sum_{j=1}^{i-1} \frac{a_{i}^{1} a_{j}^{2}+b_{i}^{1} b_{j}^{2}}{2} \int_{[k \delta,(k+1) \delta]} \cos \left(i\left(\theta_{k}^{1}(s)\right)-j\left(\theta_{k}^{2}(s)\right)\right) d s \\
& \quad=\sum_{i=2}^{M} \sum_{j=1}^{i-1} O\left(\delta^{2}\right) O\left(\frac{1}{i j|i-j|}\right) \\
& \quad=O\left(\delta^{2}\right) .
\end{aligned}
$$

is valid. The proof of theorem is completed.

Roughly speaking, this theorem separates low-frequency error-correcting signal from parasite high-frequency oscillations. This result was known to engineers [9] for sinusoidal signals only.

This theorem, for example, allows one to compute a PD characteristic for the following typical signals [9] shown in the table hereinafter. The waveforms $f^{1,2}(\theta)$ of input signals are shown in the left diagram, and the corresponding PD characteristic $\varphi(\theta)$ is plotted in the right one.

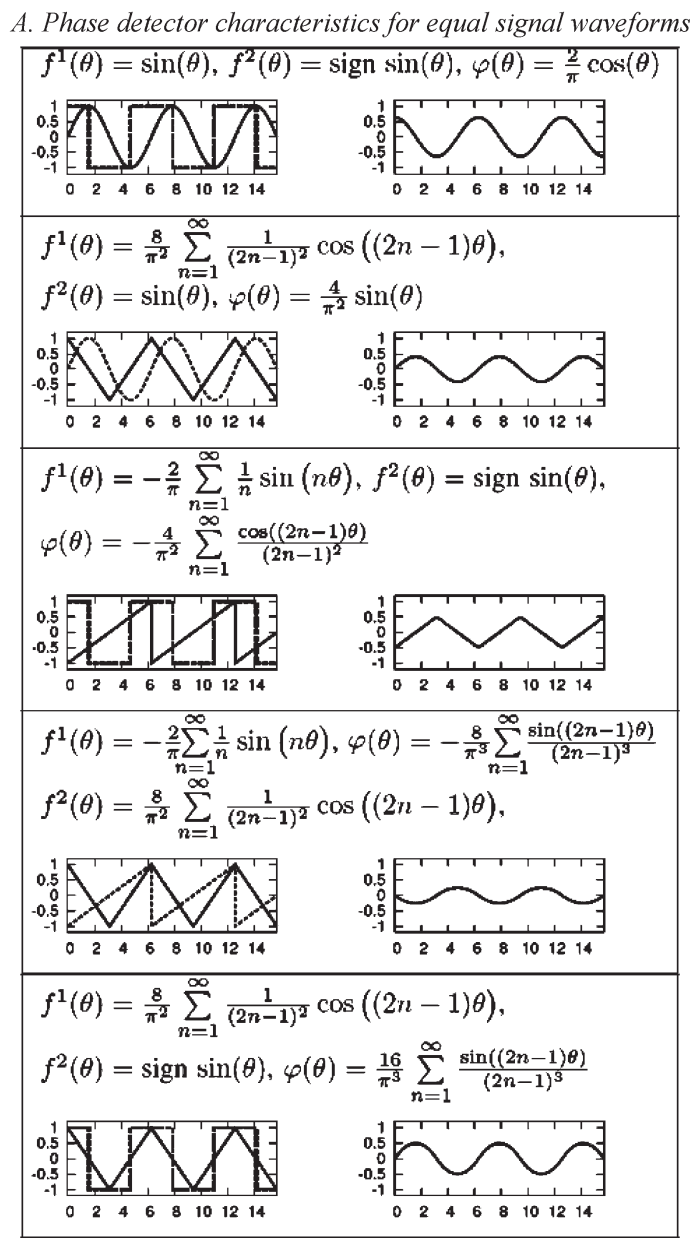

B. Phase detector characteristics for mixed signal waveforms

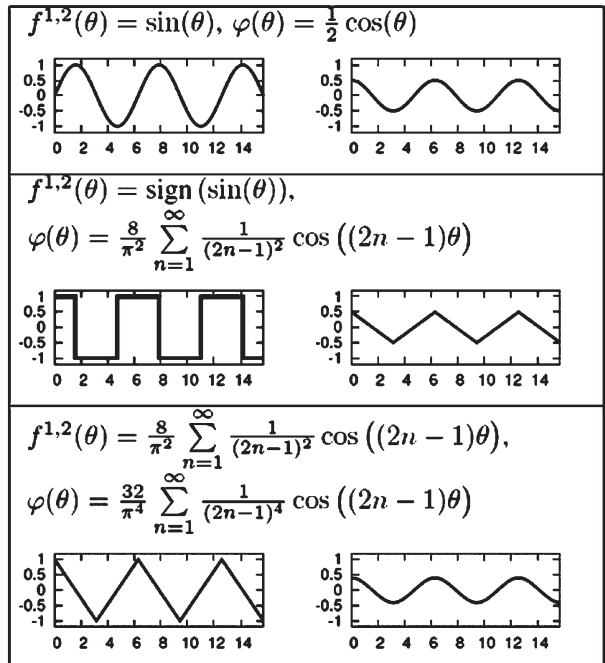

\section{DESCRIPTION OF ClassiCAl PLL in Phase-Frequency SPACE}

From the mathematical point of view, a linear filter can be described [9] by a system of linear differential equations

$$
\dot{x}=A x+b \xi(t) \quad \sigma=c^{*} x
$$

a solution of which takes the form (1). Here, $A$ is a constant matrix, $x(t)$ is a state vector of filter, and $b$ and $c$ are constant vectors.

The model of tunable generator is usually assumed to be linear [9], [10]

$$
\dot{\theta}^{2}(t)=\omega_{\text {free }}^{2}+L G(t), \quad t \in[0, T]
$$

where $\omega_{\text {free }}^{2}$ is a free-running frequency of tunable generator and $L$ is an oscillator gain. Here, it is also possible to use nonlinear models of VCO; see, e.g., [17] and [18].

Suppose that the frequency of master generator is constant $\dot{\theta}^{1}(t) \equiv \omega^{1}$. Equation of tunable generator (20) and equation of filter (19), yield

$$
\dot{x}=A x+b f^{1}\left(\theta^{1}(t)\right) f^{2}\left(\theta^{2}(t)\right) \quad \dot{\theta}^{2}=\omega_{\text {free }}^{2}+L c^{*} x .
$$

The system (21) is nonautonomous and rather difficult for investigation [4]. Here, Theorem 1 allows one to study more simple autonomous system of differential equations [in place of nonautonomous (21)]

$$
\begin{aligned}
\dot{x} & =A x+b \varphi(\Delta \theta) \quad \Delta \dot{\theta}=\omega_{\text {free }}^{2}-\omega^{1}+L c^{*} x \\
\Delta \theta & =\theta^{2}-\theta^{1} .
\end{aligned}
$$

Well-known averaging method [19]-[21] allows one to show that solutions of (21) and (22) are close under some assumptions. Thus, by Theorem 1, the block scheme of PLL in signal space (Fig. 1) can be asymptotically changed [for high-frequency generators, see conditions (2)-(4)] to the block scheme on the level of phase-frequency relations (Fig. 3).

In Fig. 3, PD has the corresponding characteristics. Thus, using asymptotic analysis of high-frequency oscillations, the characteristics of PD can be computed. Methods of nonlinear analysis for this model are well developed [4]. 


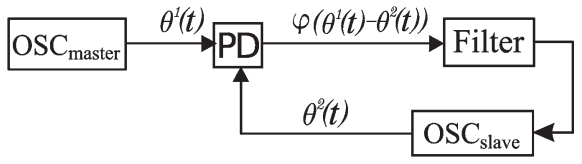

Fig. 3. Block scheme of PLL in phase-frequency space.
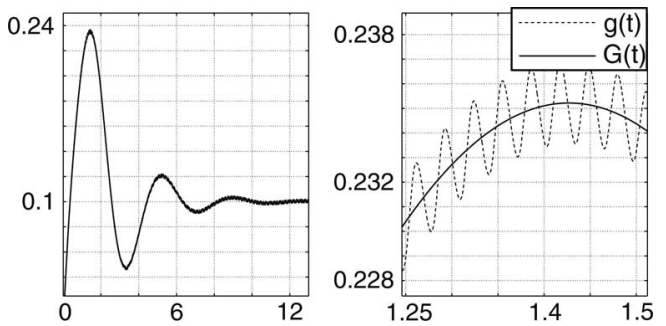

Fig. 4. $\omega_{\text {free }}^{2}=99 \mathrm{~Hz}, \omega^{1}=100 \mathrm{~Hz}, L=10$, filter transfer functions $1 /(s+1)$, and triangle waveforms.

The simulation approach for PLL analysis and design, based on the obtained analytical results, is discussed in [22].

It should be noted that, instead of conditions (3) and (5) for simulations of real system, one has to consider the following conditions:

$$
|\Delta \omega| \ll \omega_{\min } \quad\left|\lambda_{A}\right| \ll \omega_{\min }
$$

where $\lambda_{A}$ is the largest (in modulus) eigenvalue of the matix $A$. Also, for the correctness of transition from relation (8) to relation (12), one has to consider $T \ll \omega_{\min }$. Theoretical results are justified by simulation of PLL model in phase-frequency space and signal space (Fig. 4). Unlike the filter output for the phase-frequency model, the output of the filter for signal space PLL model contains additional high-frequcy oscillations. These high-frequency oscillations interfere with efficient qualitative analysis and simulation of PLL.

The passage to analysis of autonomous dynamical model of PLL (in place of the nonautonomous one) allows one to overcome the aforementioned difficulties, related with modeling PLL in time domain, which were noted in a survey lecture of well-known American specialist D. Abramovitch at American Control Conference, 2008: One has to simultaneously observe "very fast time scale of the input signals" and "slow time scale of signal's phase."

\section{CONCLUSION}

The approach, proposed in this brief, allows one (mathematically rigorously) to compute multiplier PD characteristics in the general case of signal waveforms and to proceed from analysis of classical PLL in time space to analysis and simulation in phase-frequency space. This allows one to effectively simulate classical PLL.

\section{REFERENCES}

[1] G. A. Leonov, N. V. Kuznetsov, and V. I. Vagaitsev, "Hidden attractor in smooth Chua systems," Phys. D, Nonlin. Phenom., vol. 241, no. 18, pp. 1482-1486, Sep. 2012.

[2] N. A. Gubar, "Investigation of a piecewise linear dynamical system with three parameters," J. Appl. Math. Mech, vol. 25, no. 6, pp. 1519-1535, 1961.

[3] A. Viterbi, Principles of Coherent Communications. New York: McGraw-Hill, 1966.

[4] J. Kudrewicz and S. Wasowicz, Equations of Phase-Locked Loop. Dynamics on Circle, Torus and Cylinder, ser. A. Singapore: World Scientific, 2007 , vol. 59

[5] T. J. Yamaguchi, M. Soma, M. Ishida, T. Watanabe, and T. Ohmi, "Extraction of instantaneous and RMS sinusoidal jitter using an analytic signal method," IEEE Trans. Circuits Syst. II, Analog Digit. Signal Process., vol. 50, no. 6, pp. 288-298, Jun. 2003.

[6] G. Manganaro, S. U. Kwak, S. Cho, and A. Pulincherry, "A behavioral modeling approach to the design of a low jitter clock source," IEEE Trans. Circuits Syst. II, Analog Digit. Signal Process., vol. 50, no. 11, pp. 804-814, Nov. 2003.

[7] D. Abramovitch, "Phase-locked loops: A control centric tutorial," in Proc. Amer. Control Conf., 2002, vol. 1, pp. 1-15.

[8] W. F. Egan, Frequency Synthesis by Phase Lock: John Wiley \& Sons, 2000.

[9] R. E. Best, Phase-Lock Loops: Design, Simulation and Application. New York: Mcgraw-Hill, 2003.

[10] V. F. Kroupa, Phase Lock Loops and Frequency Synthesis. New York: Wiley, 2003.

[11] G. A. Leonov, "Computation of phase detector characteristics in phaselocked loops for clock synchronization," Doklady Math., vol. 78, no. 1, pp. 643-645, Aug. 2008.

[12] G. A. Leonov and S. M. Seledzhi, "Stability and bifurcations of phaselocked loops for digital signal processors," Int. J. Bifurcation Chaos, vol. 15, no. 4, pp. 1347-1360, 2005.

[13] N. V. Kuznetsov, G. A. Leonov, S. M. Seledzhi, and P. Neittaanmäki, "Analysis and design of computer architecture circuits with controllable delay line," in Proc. 6th ICINCO, 2009, vol. 3 SPSMC, pp. 221-224, INSTICC Press, Setubal, Portugal.

[14] G. A. Leonov, N. V. Kuznetsov, M. V. Yuldashev, and R. V. Yuldashev, "Computation of phase detector characteristics in synchronization systems," Doklady Math., vol. 84, no. 1, pp. 586-590, Aug. 2011.

[15] N. V. Kuznetsov, G. A. Leonov, M. V. Yuldashev, and R. V. Yuldashev, "Analytical methods for computation of phase-detector characteristics and PLL design," in Proc. ISSCS, 2011, pp. 7-10.

[16] N. V. Kuznetsov, P. Neittaanmäki, G. A. Leonov, S. M. Seledzhi, M. V. Yuldashev, and R. V. Yuldashev, "High-frequency analysis of phaselocked loop and phase detector characteristic computation," in Proc. 8th ICINCO, 2011, vol. 1, pp. 272-278.

[17] L. Xiaolue, W. Yayun, and R. Jaijeet, "Fast PLL simulation using nonlinear VCO macromodels for accurate prediction of jitter and cycleslipping due to loop non-idealities and supply noise," in Proc. Asia South Pac. Des. Autom. Conf., 2005, pp. 459-464, ACM, New York.

[18] A. Demir, A. Mehrotra, and J. Roychowdhury, "Phase noise in oscillators: A unifying theory and numerical methods for characterization," IEEE Trans. Circuits Syst. I, Fundam. Theory Appl., vol. 47, no. 5, pp. 655-674, May 2000.

[19] N. M. Krylov and N. N. Bogolubov, Introduction to Nonlinear Mechanics. Princeton, NJ: Princeton Univ. Press, 1947.

[20] F. C. Hoppensteadt and E. M. Izhikevich, Weakly Connected Neural Networks. New York: Springer-Verlag, 1997, pp. 248-292.

[21] I. G. Malkin, Some Problems in Nonlinear Oscillation Theory. Moscow, Russia: Gostexizdat, 1962.

[22] N. V. Kuznetsov, G. A. Leonov, S. M. Seledzhi, M. V. Yuldashev, and R. V. Yuldashev, "Method for determining the operating parameters of phase-locked oscillator frequency and device for its implementation," Patent 2010149 471/08(071 509) (RU), Oct. 27, 2011. 